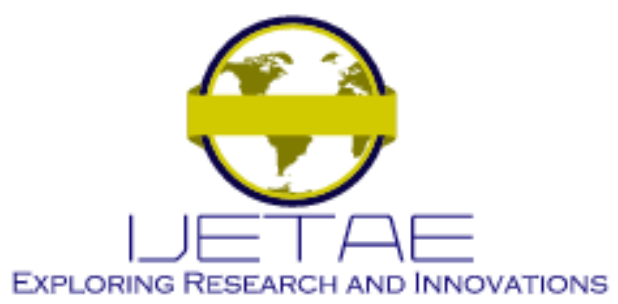

International Journal of Emerging Technology and Advanced Engineering

Website: www.ijetae.com (E-ISSN 2250-2459, Scopus Indexed, ISO 9001:2008 Certified Journal, Volume 11, Issue 06, June 2021)

\title{
Design of ALU based on Majority Logic for Low Power Applications
}

\author{
Abhishek Choubey $^{1}$, Kola Shivapriya ${ }^{2}$, Shruti Bhargava Choubey ${ }^{3}$ \\ Department of Electronics and Communication Engineering, Sreenidhi Institute of Science and Technology, Hyderabad, India
}

\begin{abstract}
Approximate calculations are a new nanotechnology paradigm for improving efficiency and reducing energy use. Most of the logic extends to many contemporary nanotechnological developments and is used for the design of digital circuits in its basic portion ( 3 input plurality, MV). This paper suggests implementations of additional compressors and ML multiplicators. An additional bit discovery circuit is used for the proposed compressors. The size of the multiplier is calculated by a control factor for the importance of different extra bits. The designs proposed are tested with hardware (for example, time frame and port complexity) as well as with error calculation. These designs have superior performance in terms of area and delay. The validity of the proposed designs is also shown by case tests of the error resistance implementation.
\end{abstract}

Keywords - Rationale for majority, rough adder, rough generator, parts for supplements, rough compressors,

\section{INTRODUCTION}

The power dissipation for the integrated circuit (IC) architecture is one of the main issues to achieving high performance. The integration of error-tolerant computing errors, including pattern recognition, machine learning and multimedia signal processing, [1-2], is a very promising way of minimizing energy use and reliability in circuits and devices [1-2]. Approximate arithmetic circuits in computers is commonly explored using CMOS technology. Prototypes for approximate manufacturers, multipliers, and dividers [3-6] have been created in both floating-point and fixedpoint formats. Error metrics including the average error gap and the proposed study of the errors of the Relative MED (RMED) and Normalized MED (NMED) and the estimated arithmetic circuits operations were suggested [7]. The technologies approaching CMOS have been suggested for the completion of the Moore law [11], with the latest developments in nanotechnologies (NML)[10] and spinwave systems (CMOS). As CMOS comes close to its technical constraint. All of the technology is built on a plurality logic that differentiates it as a digital interface framework from conventional Boolean logic.
A logical multi-input function is shown in figure 1 which executes the main door; three-input plurality door (voter, MV) logical expressions are provided by

$$
F=M(X, Y, Z)=X Y+X Z+Y Z
$$

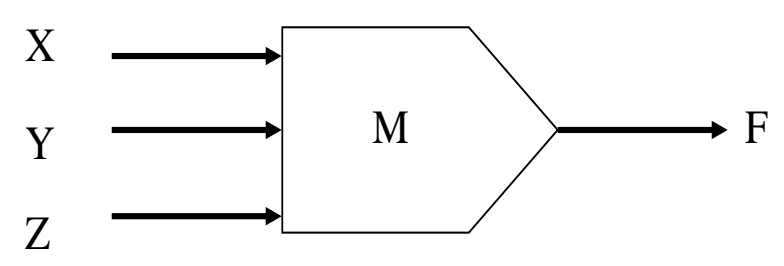

Figure 1 Majority gate for 3-input

significant improvement in power consumption is predicted from the application of approximate computing to Modern Nanotechnologies. However, due to the different underlying logics, it is explicitly impossible to construct approximate CMOS circuits; few designs have been examined of approximate circuits with ML [12-15]. An approximate 1-bit full adder(AFA) was proposed but there was no identity of available multi-bit designs. In [13], considering 1-bit and multi-bit AFAs a variety of AFAs based on ML were proposed. The Estimated Multiplier (AM) was based on 4:2 compressor. Multiplier in image recognition. Two theoretical one-bit compressors based on AFA[12], 4:2 were proposed. [15] Both ML-based AMs and ML-based AFAs were described in this paper. The MLAFA has multi-bit MLAFAs which combine one-bit MLAFAs. Furthermore, a two-bit MLAFA is designed to reduce logic and be more accurate. The 2/2-size MLAM with extra bits is suggested. The complementary bit scope can also be evaluated in relation to the multiplier size; by adding the so-called impact variable, the value of different additional bits can be evaluated. Little estimated ML Compressors are built to MLAFA (MLACs). Or to simplify K-Map; then the circuit reduces it. A review and a hardware test confirm the proposed designs. Case tests with possible image processing adders and multipliers are also used as portion of this calculation. 


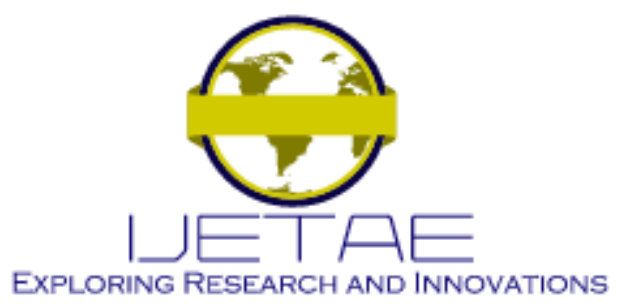

International Journal of Emerging Technology and Advanced Engineering

Website: www.ijetae.com (E-ISSN 2250-2459, Scopus Indexed, ISO 9001:2008 Certified Journal, Volume 11, Issue 06, June 2021)

This article has been expanded from the preceding conference [13]. Big variations are suggested: A $2 \times 2$ MLAM, with additional bits included; a new analysis is given for the selection of additional bits; a new two-bit MLAFA, based on the reductions in realistic table; can be used for multibit adder design; K-Map and 1-bit MLAFA simplification is suggested exact and precise; It is proposed approximate circuits reduction by 4 to 4 and 8 to 8 MLAM pipelines; case studies have been proposed for the treatment of photos by the application of MLAFAs and MLAM's.

\section{BACKGROUND}

\section{A. ML-based approximate design}

The proposed MLAFA one-bit (MLAFA1) was presented in [12]. (Fig. 2). The $X, Y, Z$ are inputs and Sum and Carry are the outputs. MLAFA1 manages output Sum as a supplement to Carry; 2 errors are invoked (including 8 input combinations), but two important one inverter and gates are spared. The inputs in the true table show how the MLAFA outputs are different from the full adder (EFA).

Carry= $M(X, Y, Z)$

Sum $=$ Carry

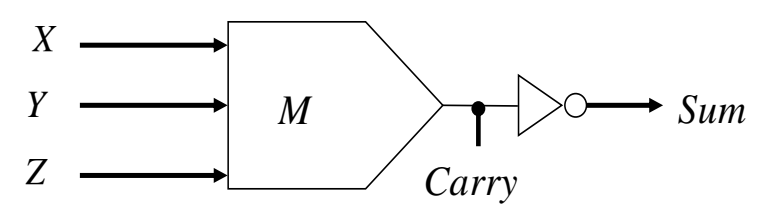

Figure 2 MLAFA1 diagram

\section{B. 4:2 Compressor based on ML Approximate}

A compressor is a significant component of a multiplier. Let $Q_{5}, Q_{4}, Q_{3}, Q_{2}, Q_{1}$ and $S$, disconnect and execute outputs be used; there are two full compressors for a 4:2 compressor serially linked [16]. It was proposed to use a reality table-3 [14] to use approximately $4: 2$ compressor (MLAC1) (Fig. 3). MLAC 1 input Q5 output is the same in 24 out of 32 cases as the performance logic and Cout has the same value as $Q 4$ in 24 of 32 cases. This alters the amount of the error reduction contribution. The resulting equations are

$$
\begin{aligned}
& \text { Carry }=Q_{4} \\
& \mathrm{Cin}=Q_{5} \\
& \mathrm{~S}=\mathrm{M}\left(Q_{2}, Q_{3}, \mathrm{M}\left(Q_{4}, Q_{5}, Q_{1}\right)\right)
\end{aligned}
$$

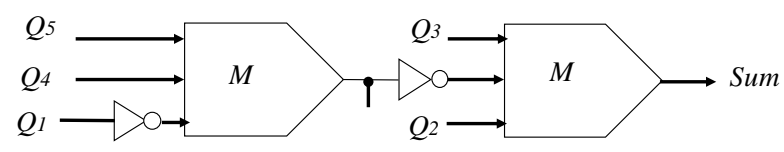

Figure 3 MLAC1 diagram [13]

In order to expand accuracy, one bit MLAFA1 is substituting for the other EFA in MLAC3. All of the proposed designs depend on three-input majority doors, although similarities in the article are not taken into account in MLAC3. The 5-input majority barrier for MLAC3 is needed.

\section{Error Metrics}

Where approximate numerical mistakes are made, metrics calculate the exactness of approximate circuits. In this article, we use the standardized mean distance error to evaluate the designs (NMED). The standardized mean error path is abbreviated as NMED. The mean (MED) error is the absolute difference between all potential inputs projected and accurate effects. Maximum Absolute Error (MAE) is an acronym for Maximum Absolute Error. The following are outlined: ED, MAE MED, NMED:

Table 1

Error Metrics

\begin{tabular}{|c|c|}
\hline MED & PED N \\
\hline MAE & $\max \{$ ED $\}$ \\
\hline NMED & MED MAX \\
\hline ED & $\mid$ ExR-ApR $)$ \\
\hline
\end{tabular}

When ApR, ExR, MAX and $\mathrm{N}$ report the exact results, the approximate results, the number of possible inputs and the total value of the output. The MLAFA 1-bit (MLAFA2) and the EFA1 1-bit (12) respectively are proposed in this section. A 2-bit architecture reduction scheme is used in the second approach. and the second method is a 2-bit truth panelbased technique. The first approach compares the proposed 1-bit MLAF as with the previous one. In addition, the proposed prototypes are drawn up in cascade by multi-bit MLAF as.

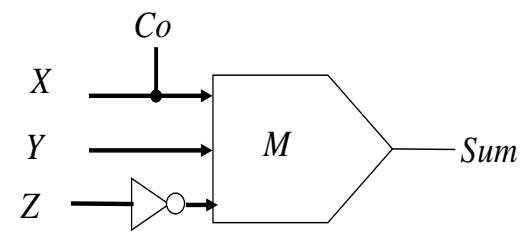

Figure 4 MLAC1 diagram 


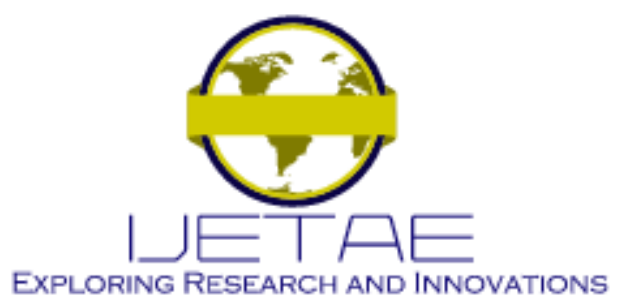

International Journal of Emerging Technology and Advanced Engineering

Website: www.ijetae.com (E-ISSN 2250-2459, Scopus Indexed, ISO 9001:2008 Certified Journal, Volume 11, Issue 06, June 2021)

\section{MODIFIED APPROX IMATE FULL ADDER}

\section{A Approximate full adder}

The modified approximate full adder is shown in figure 4.The sum expression of modified approximate adder is shown in equation (7)

$\operatorname{Sum}=\mathrm{M}\left(\mathrm{C}_{0}, \mathrm{M}(X, Y, Z, Z)=\mathrm{M}(X, Y, Z)\right.$

Table 2

Truth table of MLAC1

\begin{tabular}{|l|l|l|l|l|}
\hline \multicolumn{2}{|c|}{ Inputs } & \multicolumn{2}{c|}{ MLAC1 } \\
\hline \multicolumn{1}{|c|}{$Y$} & \multicolumn{1}{c|}{$Z$} & \multicolumn{2}{c|}{$S$} & $C_{0}$ \\
\hline 0 & 0 & 0 & 0 & 0 \\
\hline 0 & 0 & 1 & 1 & 0 \\
\hline 0 & 1 & 0 & 0 & 1 \\
\hline 0 & 1 & 1 & 1 & 0 \\
\hline 1 & 0 & 0 & 0 & 1 \\
\hline 1 & 0 & 1 & 1 & 0 \\
\hline 1 & 1 & 0 & 0 & 1 \\
\hline 1 & 1 & 1 & 1 & 1 \\
\hline
\end{tabular}

The MLAFA2,EFA and MLAFA1[12], and the suggested are compared to Table 2 about the number and the number of gates (MV), NMED, inverter number (INV), and latency in the transport (D) and delay (Dcarry). Just the number of majority gates normalizes retards in ML-based nanotechnologies (as measured in this paper). This does not require the pause of the converters.

It is often very weak relative to ML gate [17] [9]. In contrast to EFA, MLAFA2 saves two major gated doors, an inverter, and one wait. MLAFA2 reduces transportation delay to 0 as compared to MLAFA1[12], reducing the critical trail length for large-scale designs. Though Cout (which can be propagated at higher bits) is a significant error in the proposed MLAFA2, MLAFA1[12], and MLAF A2 cascades, they are less than the MLAFA1[12] cascade.
Table 3

1-Bit comparison

\begin{tabular}{|c|c|c|c|c|c|c|}
\hline $\begin{array}{c}\text { Type of one-bit } \\
\text { adder }\end{array}$ & $\begin{array}{c}\text { NME } \\
\mathrm{D}\end{array}$ & MAE & D & Dcarry & MV & INV \\
\hline MLAFA2 & .083 & 1 & 1 & 0 & 3 & 2 \\
\hline MLAFA1[10] & .083 & 1 & 1 & 1 & 1 & 1 \\
\hline EFA & 0 & 0 & 2 & 1 & 1 & 1 \\
\hline
\end{tabular}

In this segment, two approaches to 2-bit MLAFAs are suggested. As proposed (hybrid nature), the first principles merge MLAFA2 with MLAFA1; the second method uses a framework for establishing reality for 2-bit MLAFA. The inputs of the 2-Bit Adder are given with the $\mathrm{Cin}=\mathrm{a} 1 \mathrm{a} 0$, $\mathrm{B}=\mathrm{b} 1 \mathrm{~b} 0$, and the outputs are $\mathrm{S}=\mathrm{s} 1 \mathrm{~s} 0$. 3.2.1 2-bit hybrid based on MLAFA for 2-bit MLAFAs, a cascade is considered 2 MLAFAs (MLAFA1 and MLAFA2), with four different configurations; The MLAFA11 2-bit architecture generated MLAFA1 cascaded. In MLAFA2 setup, the MLAFA2 cascade also results with MLAFA2. MLAFA12 consists of both MLAFA1 and MLAFA.

\section{B Two-bit MLAFA (for 2 operands)}

$\mathrm{X}$ and $\mathrm{Y}$, there are different alternate combinations. $\mathrm{X}=$ 00 or $Y=00$ and $X=11$; and $Y=11$ are not considered to be of low complication when the Gaussian picture processing distribution is included for the true table. The reduced table are shown in Table 3.

Comparison and discussion Approximate hybrid supplements MLAFA1 and MLAFA2 was introduced in 14 out of 32 input cases defects. There are flaws in 16 of 32 inputs in the design for the truth table reduction. Table 3 shows the NMED and MAE for these MLAFAs. MLAFA22 has the best delay performance possible; due to inverters, multibit design error is of higher importance. In comparison, two hybrid MLAFAs with the same one-bit Two different one-bit MLAFA variants have more errors than MLAFAs. Taking the amount of MLAFA12 needs a smaller inverter than MLAFA21; the latency of MLAFA21 is less than MLAFA12. There are two more main gates in relation to other 2-bit MLAFAs are needed for MLAFA33, but in contrast to MLAFA12 and MLAFA21, NMED is reduced by 10 percent.

\section{Submitted Multifunctional MLAFAs}

For 2 bit cascading MLAFAs in this segment, multibit MLAFAs (including 4 and 8 bits models) are considered. 


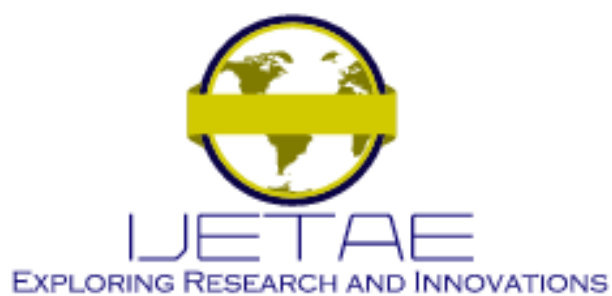

International Journal of Emerging Technology and Advanced Engineering

Website: www.ijetae.com (E-ISSN 2250-2459, Scopus Indexed, ISO 9001:2008 Certified Journal, Volume 11, Issue 06, June 2021)

Consider MLAFA 4-bit for $\mathrm{A}=\mathrm{a} 3 \mathrm{a} 2 \mathrm{a} 1 \mathrm{a} 0, \mathrm{~B}=$ b3b2b1b0, Cin and $\mathrm{S}=\mathrm{s} 3 \mathrm{~s} 2 \mathrm{~s} 1 \mathrm{~s} 0, C_{4}$ for defined outputs. Like the planned two-bit hybrid MLAFA, 2-bit MLAF as can be cascaded on four-bit MLAFA's . A delay of up to 50 percent is increased. Since the MED/NMED is the most important advantage of the lower gate number and latency. MLAFA1221 has a number of advantages. The MED/NMED for MLAFA2121 and MLAFA211 is the same, but MLAFA2121 takes substantially less time. With a MED decrease, MLAFA1212 requires one fewer inverter than MLAFA2112.. MLAFA2121 is the safest architecture since it reduces the number of doors and delays by 67 percent.

For selective use of hardware from table 3, the NMED is less than 0.084. MLAFA33 designs. Delays and major gates can be up to $50 \%$ more than nearly identical.

MLAFAs 8-bit is conceived by means of a cascade of two 4-bit MLAFAs to use MLAFA1212 and MLAFA212. These proposals decrease considerably the amount of doors and delays, but limit accuracy. For gates, MLAFA1212, and MLAFA1212, are lower inverters, compared to the other inverters, MLAFA1212-2121, MLAFA212-1, and MLAFA1212-21, and MLAFA1212-2121. The superior design is even heavier than the others, which makes MLAFA2121-2121 unmistakable. Main improvement 67 percent gates and 50 percent delays. Designs of LSBs processed by MLAFA1212 have major advantages. In MLAFA1212-1212, the smallest of NMEDs, we use MLAFA33 to supplement MSBs. For more accurate data. Its improves the accuracy of MLAFA33 by cutting NMED to around 0.08. Additional architecture is similar to MLAFA1212-1233 (58\% decline in majority gates and $50 \%$ delay) and MLAFA1212-333 (with a 50 percent drop in the majority and 50 percent delay). Moreover, with over 2 MLAFA33 the MAE rises. MLAFA1212-3333, MLAFA1212- Some of the architectures are also equivalent to 1233. InMLAFA1212-333, Exact Adders will replace MLAFA33 or MLAFA1212-3333 for relatively small error tolerant systems, with comparatively small errors.

\section{ML Based multiplier}

This section will cover the concept of estimated ML multiplicators based on two to two MLAMs. The so-called additional bit comes with a sorting system to compensate for mistakes. The compensation bits are added in line with the Multiplier size. Then, a compression efficient partial product reduction system (PPR) that uses precise Compression is used to a certain extent.
This is dependent on the partly supplied product (PPs) distribution and compensation bits, which allow PPs to be collected from two rows (or a low-order carry). The result will finally be calculated by the final adder.

In comparison, errors can increase markedly by increasing the degree of design, which is unacceptable in most contexts. Given the questions, one of 2 MLAM is used as one, the other is as a compensation bit, with the first period being split into two parts (denoted as 4). In this essay, we only consider one case. The same rules apply in the other situation.

If the 2/2 MLAM modules are taken into account, greater multipliers may be created, splitting the operands into many condition. In the calculation of the resulting compression, too many compensation bits lead to an increased overhead, but too few compensation bits may ultimately lead to loss of precision. Therefore, when choosing a proper number of payout bits, the balance must be measured. The compensation bits with smallest weight are overlooked when the compensation bits are selected and the mistake is rationally tested. LSBs would also impact the MSB result and not less than the MSB compensation bit overhead hardware. The LSB bits may also be omitted by deleting the MSB bits. It's the whole thing. The signed supplement bit number is shown by weight and $\mathrm{x}$ if there are multiple bits under the weight of the existing add-on bit. For example, $\mathrm{C}$ is a complementary bit(23) weight and 1 is a complementary bit(23) while 1 is only one bit weight. To measure the value of any additional Bit $\mathrm{C}_{2}$ as well as to determine the object missing to determine the impact of an additional Bit on the last NMED. The following can be defined for an n-top MLAM: Miss accuracy. Lots of accuracy. Therefore, by selecting a number of Bits of repayment, a solution has to be formed. When the compensation bits are chosen and the error governed in a fair band, the compensation bits for the lowest weight are ignored. Moreover, LSB's effect on the result of the MSB would not exceed the compensation bit's overhead hardware. As a result, the LSB restitution bits are skipped as the MSB is deleted. In this article the additional pieces are referred to denote the weight of the additional piece and $\mathrm{x}$ show the signatures. Bit where there are some extra pieces of the new weight. To measure the value of any additional Bit $\mathrm{C} 2$ ix as well as to determine the object missing to determine the impact of an additional bit on the last NMED. 


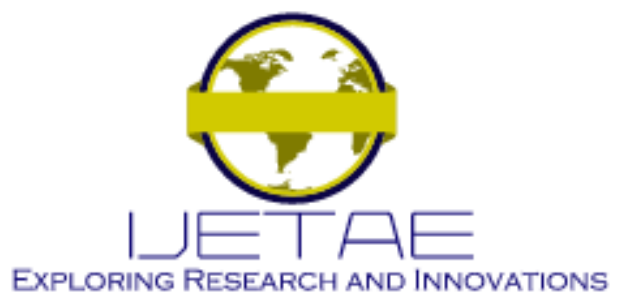

International Journal of Emerging Technology and Advanced Engineering

Website: www.ijetae.com (E-ISSN 2250-2459, Scopus Indexed, ISO 9001:2008 Certified Journal, Volume 11, Issue 06, June 2021)

E Design of MLACs

A few compressors $(P 5, P 4, P 3, P 2, P 1)$ and the outputs Sum, Cut, Carry) and K Map simplifications are presented in this section $(P 5, P 4, P 3, P 2, P 1$ are the entries, $P 2, P 1$, and Sum are the outputs). MLAFAs MLACs with a 1-bit version There are two absolute supplants in 4:2 compressors known as versions 1 and 2. Exactly 1-bit MLAFAs are substituted. Six different versions were tested using various variants of MLAFA2 and MLAFA1. The MLAFA2 is used by MLAC21 for Module 1 while MLAFA1 is used for other modules. The proposal for MLAC11 in [15]; there is two schemes for module 2 of the MLAFA2.

K-Map-based simplification It is proposed that another MLAC, namely MLAC4, minimize circuit complexity further by using two outputs to achieve a final output (rather than 3). This MLAC is designed in three phases: The following:

Step 1: Estimate of the number of outputs. To indicate the result, use 2 outputs to make binary 11 more than 11 outputs.

Step 2: approximation of K-map expression

Step 1: Simplified formulas can be described by adding a few defects to the majority logic properties.

Phase 3: To measure error distance, combine step 1 and step 2. Phase 1 displays an approximate K-Map resulting in seven failures. It's shortened bit length. the final articulated expression is therefore conceivable.

\section{RESUlt DisCUSSION}

\section{A One bit result comparison}

In terms of consumption and latency in most gates, the 1-bit MLAFA designs were similar. The MEDs for NMDs are MLAC2[15], MLAC22-2, MLAC12-1 25\% smaller, with MLAC22-1 and MLAC12-1 delays less than those of MLAC2[15], and the MEDs of the MEDs are 50\% smaller than the other designs. This is MLAC12-shortest 1's time. When the output is connected to the next device, the total delay can be reduced. as a result of which MLAC22-2.

The MLAFA efficiency of the MLAC12-1 is the best of all. The proposed method involves 67 percent of the doors, 50 percent of the inverters, 50 percent of delays, and 67 percent of the transport chain were saved, despite the exact configuration. For MLAC1[14], no delays in the transport chain are needed, and the proposed projects achieve a reduction in NMED of up to $25 \%$.
When the simplification based on the K-map is planned, the delay is slightly lower than the above designs, and the bug (MAE and NMED) is slightly higher. MLAC4 requires two additional three-input majority gates, but only two outputs instead of one-bit MLAFAs. It does not make use of an inverter. It also saves $33 \%$ of the largest Ports, both inverters, $50 \%$ delays and $33 \%$ of the carriage chain, compared to the accurate specification.

MLAM Circuitry architecture proposed for PPR It uses the PP distribution to shorten the critical path length during compression. This architecture in order to achieve effective and rough compression, the powerful PPR architecture is studied using the pipeline of 4 to 4 and 8 MLAM architectures, with some additional bits (or a carry in the lowest order). Besides the above solution, there is another arrangement for an 8x8 MLAM setup, in which there are 4 different versions. 4 MLAMs 4.4.1. PP compression that is precise (EC) For compression, one-bit adders are utilized. Based on the quantity of extra bits, four distinct compression algorithms are available. PPs are a solid line arithmetic feature. There is no difference in the number of extra bits. To measure the result separately from the set of the supplementary parts, a 4-bit adder is needed. The difference is the numeral of one-bit compression supplements used.

Approximate compression (AC) in the reduction circuit, the proposed MLAFAs and compressors should be used to minimize hardware and delays. In PPs with dotted lines, approximate arithmetical components are shown. $\mathrm{P} 1$ is used by the compressors. In arithmetic circuits, the pcs compact on the right side of the medium line in terms of delays (they have the same delay as the left compression circuits). Two one-bit EFAs are selected to better track mistakes (rather than 2-bit MLAFA). If an estimated compressor is used with 2 outputs, a 3-bit half adder is needed for the end result. A compressor with approximate 3 outputs requires a full 3-bit adder otherwise. However, as $\mathrm{p}$ rises, errors rise; a new approach is then recommended to higher $\mathrm{p}$ values.

\section{B Discussion of four to 4 MLAMs}

The various circuitry designs of PPRs are compared in table 4 to the exact designs from [23-24]. The MLAFA2, MLAC22-2, MLAC21 or MLAC4 proposed was used for approximate compression. Approximate compression reduces accuracy but reduces the total compression hardware. If $q$ is larger, roughly compression is less pronounced. 


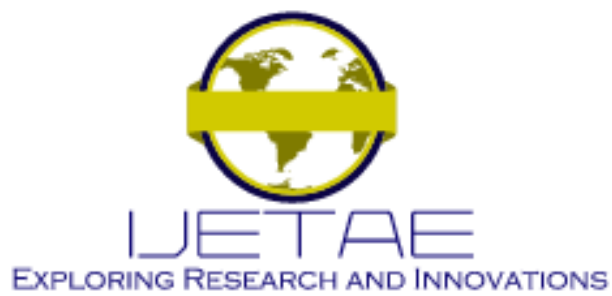

International Journal of Emerging Technology and Advanced Engineering

Website: www.ijetae.com (E-ISSN 2250-2459, Scopus Indexed, ISO 9001:2008 Certified Journal, Volume 11, Issue 06, June 2021)

When $q$ is 1 , rough compression has a considerable effect on MAE and NMED but when $\mathrm{p}$ is 4 , rough compression only has a 7.5 percent effect on NMED and only a 24 percent effect on MAE. When $\mathrm{q}$ is $4,4 \times 4$ MLAM is used, as reported. A slight increase of NMED and MAE has been the result of AC2 $(q=4)$. This means, that for an algorithm which can accommodate relatively huge errors a greater approximation should be used. When $q$ is similar to 1 , the proposed MLACs have shown excellent performance, which enhances their overall accuracy by adding just a few majorities. If additional Bits are required in order to be 4 to $4 \operatorname{MLAM~AC2~(q=1),~it~not~}$ only reduces overhead hardware and late hardware, but also contributes to more downtime and latency. In contrast with the particular designs from [21-24], the proposed design suggestively reduces overhead and hardware latency. For example, $4 \%$ of all MLAM-AC2 ( $q=1)$ who use MLAC222 keeps at least $37 \%$ of major portals and $46 \%$ of the inversion counter the time lag is 50 percent. MLAMs Eight ore eight MLAMs Using four MLAMs 4 to 4 MLAMs can be designed as an 8 MLAM module, which enables the development of 4 . The product of 4 to 4 MLAMs is 8 bits regardless of the additional bits and the PPR circuit selection. $N$ The amount of extra bits) is possible; but, as in Section, additional bits of MSBs might be available The hardware reduction is not the main objective of missed or assigned to LSBs under this arrangement. It is only likely that $4=4$ MLAMs are better used with 4 compression MLAMs in the previous work. When $\mathrm{q}$ is close to 1 the multiplier 4-per-4 MLAM-AC2 $(\mathrm{q}=1)$ gives a stronger outcome with approximate compression. Exact compression 4-5 MLAMAC1 $(q=1)$ is the performance in the NMED, MAE, and hardware and the delay. In comparison with a multiplier, the latter is better than all the other instances4=4 MLAM-AC compression $(q=2)$ MLAM-AC compression $(\mathrm{q}=3)$ with an approximate compression of MLAMAC compression $(q=1)$.

\section{C $8 \times 8$ MLAMs Using two MLAMs}

A $2 \times 2$ MLAM module enables the construction of all PPs to be manufactured simultaneously and has less clock cycles compared to $4 \times 4$ MLAMs. In both cases, there are just 1-bit adders. The decrease of Ry $p$ increases the hardware required; compression requires just 3 stages when $\mathrm{p}$ is greater than 10. Four compression phases are necessary, otherwise; The pause is not affected by extra bits. As shown, $\mathrm{p}=10$ will be tested for estimated MLAFA2 and MLAC PPR circuits. Compression with MLACs and the new version with 2-piece MLAC is evaluated.
In comparison, the circuitry specification of approximate PPR is 8 to 8 MLAMs. Compared to the precise compression, hardware and latency were greatly reduced as stated earlier. Approximate compression not only decreases the time, but also the number of key doors with a small reduction of precision reduces by more than $13 \%$. The usage of MLAC4 Moreover Decreases the needed hardware but also reduces inaccuracies, since the activities are less calculated, which ensures the best overall efficiency.

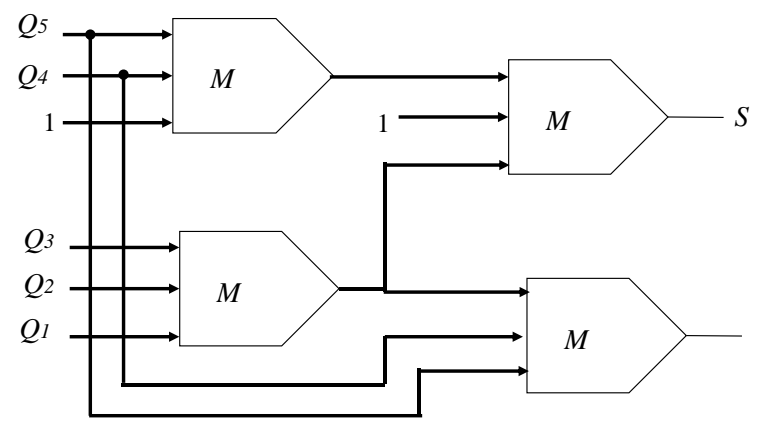

Figure 5 MLCA4 diagram

The top designs are chosen using MLAM and compared to the actual designs from [24]. The number of key doors decreases by at least $25 \%$, the inverter numbers by $27 \%$, and the latency by $33 \%$ in comparison with exact designs, by the 4 th to the 4th MLAM-C2 (p=1) with M LAC22. The 4-out of 4 multipliers prototypes are not as good as the 2out of 2. The circuitry is considerably decreased without major errors for designs using two or two multipliers. The MLAC4 architecture cuts the number of main doors by as many as $48 \%$, up to $67 \%$, and the delays by up to $47 \%$. The 2-2 plug-in configuration cuts the number of main doors up to $50 \%$, whilst the inverters up to $53 \%$. The expectation is $47 \%$. CAD designer has been used to further test its feasibility for QCAA, as an emergent technology, with an approx. MLAC4 multiplier Architecture.

The crossing is multi-layer, and parameters were used to simulate the vector motor's coherence. Convergence tolerance: 0.00001 ; radius of effect: $55 \mathrm{~nm}$. Numbers of effect: 128000 . Default are the remaining parameters). The architectures for multipliers based on 8-bit MLAC 4 and the recent roughly CMOS (45nm) [25] are not directly comparable with the plurality logic b.

In separate parts of the suggested scheme are housed the VERILOG HDL shown in the simulator and FPGA Synthesis Xilinx ISE; MLA ALU schematic RTL: MLA M scheme: 


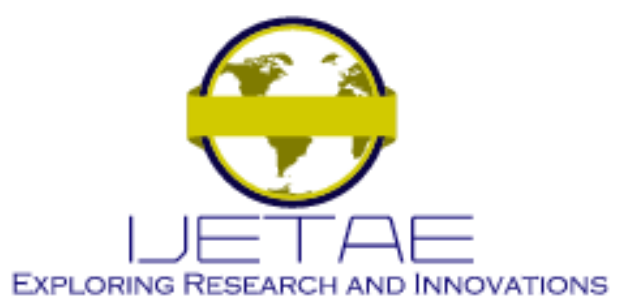

International Journal of Emerging Technology and Advanced Engineering

Website: www.ijetae.com (E-ISSN 2250-2459, Scopus Indexed, ISO 9001:2008 Certified Journal, Volume 11, Issue 06, June 2021)

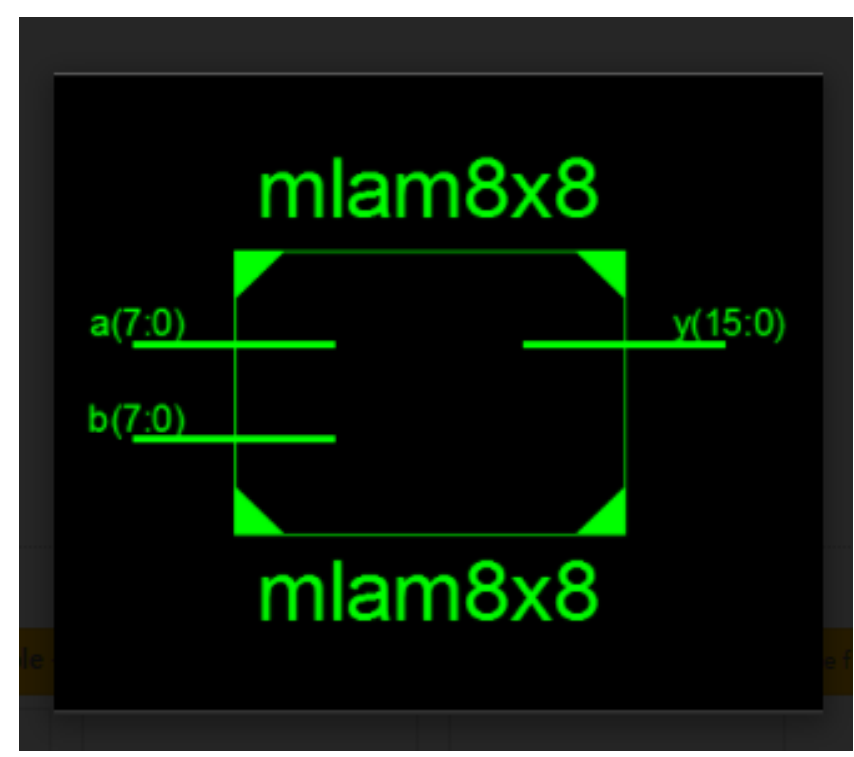

Figure 6 Schematic of MLAM $8 \times 8$ multiplier

Table 4

Simulation results

\begin{tabular}{|c|c|c|}
\hline 2-bit Multiplier & Area $(\boldsymbol{\mu m})$ & Delay $(\mathbf{n s})$ \\
\hline Array Multiplier & 40 & .98 \\
\hline MLAM2 & 30 & .97 \\
\hline MLAM 2×2 & 25 & .83 \\
\hline 4-bit Multiplier & \multicolumn{2}{|l}{} \\
\hline Array Multiplier & 210 & 3.24 \\
\hline MLAM 4×4 & 120 & 2.65 \\
\hline 8-bit Multiplier & \multicolumn{2}{|}{} \\
\hline Array Multiplier & 430 & 6.62 \\
\hline MLAM 8×8 & 300 & 5.34 \\
\hline
\end{tabular}

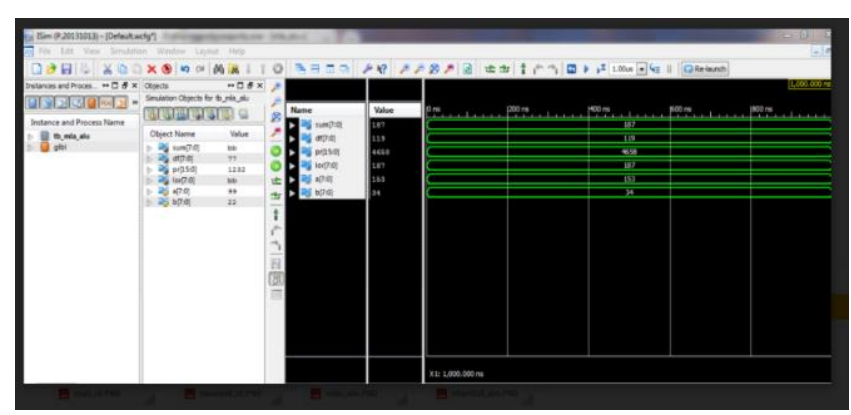

Figure 7 Simulation results of proposed multiplier

\section{CONCLUSION}

A plan, analysis and evaluation by estimated suppliers and approximate multipliers based on majority thinking has been presented in this report. It is suggested that ML-based one-bit, two-bit and multibit AFAs should be used, which show lower device complexity and a lower time than the exact counterpart but are only slightly precise. By incorporating the 'complement bits' with the ML multi-bit AM's, which combine some approximate (such as the suggested MLACs and approximate PPR circuits): A factor of impact was developed in order to calculate the value for different complement bits; the complement bits were thoroughly analyzed. Various MLACs based on the simplification of MLAFAs or K-Map have been suggested and used, depending on the multiplier dimension, in approximately 8 to 8 MLAM PPR circuitry designs.

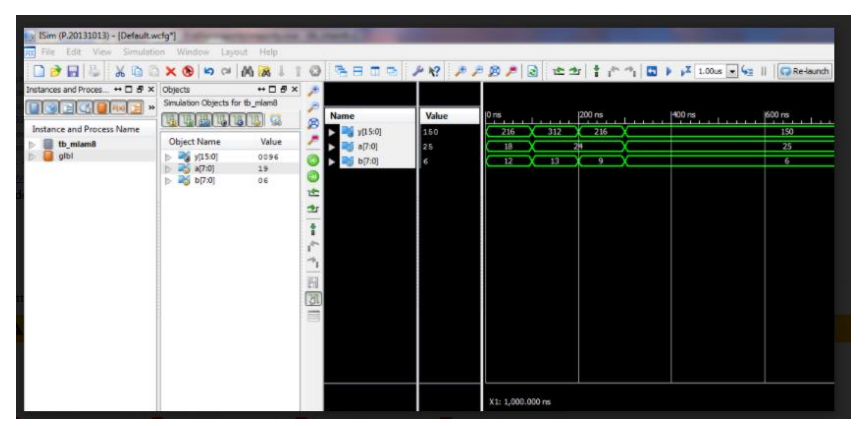

Figure 8 Simulation results of proposed multiplier

The conclusions can be reached. (1) The new 1-bit MLAFA with a big flaw can be compared to the multi-bit MLAFAs. A response to improved accuracy is the MLAFA33 initiative that aims to minimize portals and 50\% delay) and MLAFA1212-333 for eight-bit supplements are preferable (a 50 percent reduction in plurality gates and a 50 percent reduction in delay). (2) The 2 The 2Multi-bit multipliers can be designed selectively by placing additional bits to 2 MLAM. The theoretical study found that with NMED from 3 to 4 the $\mathrm{p}$ multiplier increases dramatically, with NMED increasing steadily if $\mathrm{p}$ is less than 10 with the $\mathrm{p}$ multiplier from 8 to 8 , and NMED grows slowly if $p$ is larger than 10 . Thus, $q=3$ and $q=10$ are the most appropriate alternatives for the four multipliers by four and the eight multipliers by eight. (3) The MLAC 22-2 and MLAC12-1 have the best overall results of those MLAFs. $67 \%$ of majority gateways, $50 \%$ inverter, $50 \%$ waits and $67 \%$ carriages are spared on the proposed version compared with the exact version. 


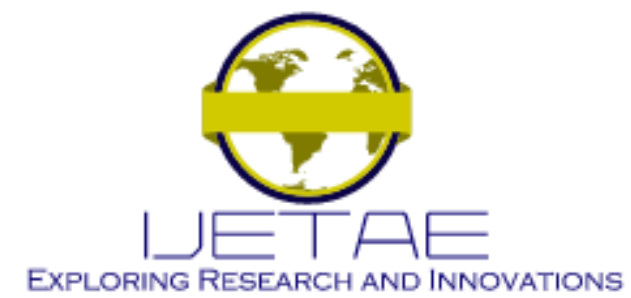

International Journal of Emerging Technology and Advanced Engineering

Website: www.ijetae.com (E-ISSN 2250-2459, Scopus Indexed, ISO 9001:2008 Certified Journal, Volume 11, Issue 06, June 2021)

The string styles. Although there are only 2 MLAC4 outputs that require two additional majority gates for 3 inputs, MLAC4 will result in an expected decrease in PPR activity. The $2 / 2(\mathrm{p}=10)$ plug based on MLAC4, an 8-to-8 plug, decreases the number of the major doors by up to $48 \%$, up to $67 \%$ and the latency by up to $47 \%$, thereby greatly decreasing hardware without major errors.

\section{REFERENCES}

[1] S. L. Lu, "Speeding up processing with approximation circuits," Computer, vol. 37, no. 3, pp. 67-73, 2004.

[2] J. Han and M. Orshansky, "Approximate computing: an emerging paradigm for energy-efficient design," in Proc. European Test Symposium, pp. 1-6, 2013

[3] S. Hashemi, R. Bahar, and S. Reda, "DRUM: A Dynamic Range Unbiased Multiplier for Approximate Applications," in Proc. IEEE/ACM International Conference on Computer Design, pp. 418 425, 2015.

[4] V. Gupta, D. Mohapatra, A. Raghunathan, and K. Roy, "Low-power digital signal processing using approximate adders," IEEE Trans Computer-Aided Design of Integrated Circuits and Systems, vol. 32, pp. 124-137, 2013.

[5] S. Rehman, W. El-Harouni, M. Shafique, A. Kumar, and J. Henkel, "Architectural-space exploration of approximate multipliers," in Proc. Int. Conf. Computer-Aided Design, pp. 1-6, 2016.

[6] A. Momeni, J. Han, P. Montuschi, and F. Lombardi, "Design and analysis of approximate compressors for multiplication," IEEE Transactions on Computers, vol. 64, no. 4, pp. 984 - 994, 2014.

[7] J. Liang, J. Han, and F. Lombardi, "New metrics for the reliability of approximate and probabilistic adders," IEEE Trans Computers, vol. 62, no. 9, pp. 1760-1771, 2013.

[8] K. Walus and G. Jullien, "Design tools for an emerging SoC technology: quantum-dot cellular automata," in Proc. IEEE, vol. 94, no. 6, pp. 1225-1244, 2006.

[9] C. Lent and P. Tougaw, "A device architecture for computing with quantum dots," in Proc. IEEE, vol. 85, no. 4, pp. 541-557, 1997.

[10] M. Vacca, M. Graziano, J. Wang, F. Cairo, G. Causapruno, G. Urgese, A. Biroli, and M. Zamboni, "Nanomagnet logic: an architectural level overview," Lecture Notes in Computer Science, pp. 223-256, 2014.

[11] A. Khitun and K. L. Wang, "Nano scale computational architectures with spin wave bus," Superlattices and Microstructures, vol. 38, no. 3, pp. 184-200, 2005.

[12] C. Labrado, H. Thapliyal and F. Lombardi, "Design of majority logic based approximate arithmetic circuits," in Proc. IEEE Int. Symp. Circuits and Systems, pp. 2122-2125, 2017.
[13] T. Zhang, W. Liu, E. McLarnon, M. O'Neill and F. Lombardi, "Design of majority logic (ML) based approximate full adders," in Proc. IEEE Int. Symp. Circuits and Systems, 2018.

[14] M. H. Moaiyeri, F. Sabetzadeh, and S. Angizi, "An efficient majority-based compressor for approximate computing in the nano era," Microsystem Technologies, vol. 4, pp. 1-13, 2017.

[15] S. Angizi, H. Jiang, R. F. DeMara, J. Han and D. Fan, "Majoritybased spin-CMOS primitives for approximate computing," IEEE Trans Nanotechnology, vol. 17, no. 4, pp. 795-806, 2018.

[16] C. Chang, J. Gu, and M. Zhang, "Ultra low-voltage low-power CMOS 4-2 and 5-2 compressors for fast arithmetic circuits," IEEE Trans Circuits \& Systems I, vol. 51, no. 10, pp. 1985-1997, 2004. 2168-6750 (c) 2019 IEEE. Personal use is permitted,

[17] V. Pudi, K. Sridharan, and F. Lombardi, "Majority logic formulations for parallel adder designs at reduced delay and circuit complexity," IEEE Trans Computers, vol. 66, no. 10, pp. 1824-1830, 2017.

[18] H. Cho and E. E. Swartzlander, "Adder and multiplier designs in quantum dot cellular automata," IEEE Trans Computers, vol. 58, no. 6, pp. 721-727, 2009.

[19] V. Pudi and K. Sridharan, "Low complexity design of ripple carry and Brent-Kung adders in QCA," IEEE Trans Nanotechnology, vol. 11, no. 1, pp. 105-119, 2012.

[20] S. Mittal, "A survey of techniques for approximate computing," ACM Computing Surveys, vol. 48, no. 4, pp. 62, 2016.

[21] S. L. Eddins and M. T. Orchard, "Using MATLAB and C in an image processing lab course," Proc. of 1st International Conference on Image Processing, vol. 1, pp. 515-519, 1994.

[22] P. Kulkarni, P. Gupta, and M. Ercegovac, "Trading accuracy for power with an underdesigned multiplier architecture," Proc. VLSI Design, pp. 346-351, 2011.

[23] S. W. Kim and E. E. Swartzlander, "Multipliers with coplanar crossings for quantum-dot cellular automata," Proc. 10th IEEE Conference on Nanotechnology, pp. 953-957, 2010.

[24] S. W. Kim and E. E. Swartzlander, "Parallel multipliers for quantum-dot cellular automata," Proc. Nanotechnology Materials and Devices Conference, pp. 68-72, 2009.

[25] Mohanty, B.K., Choubey, A. Efficient Design for Radix-8 Booth Multiplier and Its Application in Lifting 2-D DWT. Circuits Syst Signal Process 36, 1129-1149 (2017

[26] B. Anusha, M L Nihitha Priya , I. Ravi Teja , Abhishek Choubey "A Low Power Modified Booth Multiplier" International Journal of Emerging Technology and Advanced Engineering, Volume 10, Issue 05, May 2020

[27] Anusha, B., Nihitha Priya, M.L., Ravi Teja, I., Choubey, A. "A low power modified booth multiplier"(2020) International Journal of Emerging Technology and Advanced Engineering, 10 (5), pp. 179182.

[28] Ma, B., Wan, Z., Hu, Z., Li, Z., Han, C., Zeng, Z., Zheng, Z., Tang, T., Wu, W. "Application of simulation software in optimization of powertrain of pure electric vehicle", (2020) International Journal of Emerging Technology and Advanced Engineering, 10 (11), pp. 4255. 